\title{
Correction to: Artificial Intelligence, Brains, and Beyond: Imperial College London Neurotechnology Symposium, 2020 , by Tarasenko A, et al. BIOE 2020;2:310-313.
}

N THE SePtember 2020 issue of Bioelectricity (vol. 2, no. 3; 310-313), the article entitled "Artificial Intelligence, Brains, and Beyond: Imperial College London Neurotechnology Symposium, 2020” by Tarasenko et al. requires correction.

In the author byline on page 310, author Daryna Ivaskevych's name is misspelled.

The author byline originally states:

“Anna Tarasenko, MS, ${ }^{1}$ Mikheil Oganesyan, $M S,{ }^{l}$ Daryna Ivashkevych, MA, ${ }^{2}$ Sergii Tukaiev, PhD, ${ }^{2}$ Dauren Toleukhanov, ${ }^{\prime}$ and Nickolai Vysokov, $\mathrm{PhD}^{1,}$

The author byline should now read:

“Anna Tarasenko, MS, ${ }^{1}$ Mikheil Oganesyan, $M S,{ }^{1}$ Daryna Ivaskevych, MA, ${ }^{2}$ Sergii Tukaiev, PhD, ${ }^{2}$ Dauren Toleukhanov, ${ }^{\prime}$ and Nickolai Vysokov, PhD",

The online version of the article has been corrected to reflect this. 